\title{
Weighted pseudo almost periodic solutions to a class of semilinear integro-differential equations in Banach spaces
}

\author{
Edgardo Alvarez ${ }^{1}$ and Carlos Lizama2 ${ }^{*}$
}

\section{"Correspondence:}

carlos.lizama@usach.cl

${ }^{2}$ Departamento de Matemática y

Ciencia de la Computación,

Facultad de Ciencia, Universidad de

Santiago de Chile, Casilla 307,

Correo 2, Santiago, Chile

Full list of author information is

available at the end of the article

\begin{abstract}
In this paper we prove the existence of weighted pseudo almost periodic mild solutions for the class of integro-differential equations in the form $u^{\prime}(t)=A u(t)+\alpha \int_{-\infty}^{t} e^{-\beta(t-s)} A u(s) d s+f(t, u(t))$ where $f(\cdot, u(\cdot))$ is a Stepanov-like weighted pseudo almost periodic function and $A$ generates an immediately norm continuous $C_{0}$-semigroup on a Banach space $X$. Also, we give a short proof to show that the vector-valued space of Stepanov-like weighted pseudo almost periodic functions is a Banach space.

MSC: 35B15; 47D06; 45D05

Keywords: weighted pseudo almost periodic functions; Stepanov weighted pseudo almost periodic functions; convolution; composition; abstract Volterra equations; infinite delay
\end{abstract}

\section{Introduction}

Our concern in this paper is the equation

$$
L(u)=f
$$

where $L$ is a linear, possibly unbounded operator, and the forcing term $f$ belongs to some space of vector-valued functions, say $\mathcal{M}$. It is well known that mathematical understanding of the linear equation (1.1) is important as a preliminary critical step for the subsequent analysis of full nonlinear models. Usually, one is interested in finding conditions on the operator $L$ such that the solution $u$ belongs to the same space of vector-valued functions than $f$. Then fixed point arguments are used to obtain the desired solution of associated nonlinear problems.

We ask the following question: (Q) Can the solution $u$ be more regular that $f$ ? In other words, is it possible to find a subspace $\mathcal{N} \subset \mathcal{M}$ such that $u \in \mathcal{N}$ ?

This problem began to be studied recently and there are some cases in the literature where the answer is positive. For example, in [1], Diagana et al. solved problem (Q) taking $\mathcal{M}$ as the space of Stepanov-like weighted pseudo almost automorphic functions, $L(u):=$ $D_{t}^{\alpha} u-A u$ and $\mathcal{N}$ as the subspace of weighted pseudo almost automorphic functions. Here $A$ is a closed and linear operator defined on a Banach space $X$ and $D^{\alpha}$ denotes fractional derivative of order $\alpha>0$.

( 2015 Alvarez and Lizama; licensee Springer. This is an Open Access article distributed under the terms of the Creative Commons Attribution License (http://creativecommons.org/licenses/by/4.0), which permits unrestricted use, distribution, and reproduction in any medium, provided the original work is properly credited. 
In this paper, we are able to give an affirmative answer to $(\mathrm{Q})$ taking $\mathcal{M}$ as the space of Stepanov-like weighted pseudo almost periodic functions; $\mathcal{N}$ is the space of weighted pseudo almost periodic functions and the class of operators is defined by

$$
L(u)(t)=u^{\prime}(t)-A u(t)-\alpha \int_{-\infty}^{t} e^{-\beta(t-s)} A u(s) d s
$$

where $A$ generates an immediately continuous $C_{0}$-semigroup on a Banach space $X$. Then an application to find existence and uniqueness of mild solutions for the corresponding semilinear problem is given. The class of operators (1.2) has been studied by Ponce and the second author of this paper, in [2]. This class arises in several applied fields, like viscoelasticity or heat conduction with memory, and in such applications the operator $A$ typically is the Laplacian, the elasticity operator or the Stokes operator, among others, equipped with suitable boundary conditions. The exponential kernel $\alpha e^{-\beta t}$ is the typical choice when one consider Maxwell materials in viscoelasticity theory [3]. The authors in [2] have solved the problem of maximal regularity in several spaces of functions, i.e. starting with $f \in \mathcal{M}$ and proving that the mild solution $u$ belongs to $\mathcal{M}$. We remark that continuity fails in the case of Stepanov type functions and only measurability and integrability are required to work with this class of functions. Hence, it justifies thinking of the previous problem in the context mentioned above. To the best of our knowledge, the existence of weighted pseudo almost periodic solutions to (1.2) in the case when the forcing term $f$ is a Stepanov-like weighted pseudo almost periodic is an untreated original problem, which constitutes one of the main motivations of this work.

Weighted pseudo almost periodic solutions to classes of evolution equations have been studied since the original paper of Diagana [4] in 2006. We mention here the study of functional difference equations [5], partial neutral functional differential equations [6-8], non-autonomous differential equations [9], neutral delay equations of advanced type [10], hyperbolic evolution equations [11], partial functional differential equations [12], semilinear evolution equations [13, 14], semilinear fractional differential and integro-differential equations [15, 16], positive solutions to infinite delay integral equations [17], and second order differential equations with piecewise constant argument $[18,19]$. Recently, an interesting application to a class of discrete hematopoiesis models has been given in [20].

This paper is organized as follows. In Section 2, we first present some definitions and basic results of Stepanov-like type spaces and then we give a short and direct proof to the fact that the space of Stepanov-like weighted pseudo almost periodic functions (respectively Stepanov-like weighted almost automorphic functions) is a Banach space. In Section 3, we give sufficient conditions in order to have better regularity of Stepanov-like weighted pseudo almost periodic functions under a finite convolution with an appropriate family of bounded of linear operators defined on a Banach space $X$; see Theorem 3.3. In Section 4 we treat a semilinear problem, i.e. $f=f(t, u)$. We prove that if $f$ is Lipschitz in the second variable and Stepanov-like weighted pseudo almost periodic in the first variable, then there exists a unique mild solution in the vector-valued space of weighted pseudo almost periodic functions. See Theorem 4.7. Then, in order to have a weaker hypothesis on $f$, we prove a result of regularity for the composition of the Nemytskii operator with a Stepanov-like weighted pseudo almost periodic function, assuming a compactness condition. See Theorem 4.9. After that, we show Theorem 4.10, which gives sharper conditions 
for answering (Q). Finally, in Section 5, we present an application that illustrates the feasibility of the given hypothesis in the abstract results.

\section{Preliminaries}

In this section, we introduce some basic definitions, notations, and preliminary facts which will be used in the sequel. Particularly, we give an alternative proof to show that the space of Stepanov-like weighted pseudo almost periodic functions is a Banach space.

Throughout the paper $\left(X,\|\cdot\|_{X}\right)$ and $\left(Y,\|\cdot\|_{Y}\right)$ are complex Banach spaces and $B(X, Y)$ is the Banach space of bounded linear operators from $X$ to $Y$; when $X=Y$ we write $B(X)$.

We denote by

$$
B C(\mathbb{R}, X):=\left\{f: \mathbb{R} \rightarrow X: f \text { is continuous, }\|f\|_{\infty}:=\sup _{t \in \mathbb{R}}\|f(t)\|<\infty\right\},
$$

the Banach space of $X$-valued bounded and continuous functions on $\mathbb{R}$, with natural norm.

We recall the definitions of almost periodic and almost automorphic functions.

Definition 2.1 ([21]) A function $f \in C(\mathbb{R}, X)$ is called (Bohr) almost periodic if for each $\epsilon>0$ there exists $l=l(\epsilon)>0$ such that every interval of length $l$ contains a number $\tau$ with the property that

$$
\|f(t+\tau)-f(t)\|<\epsilon \quad(t \in \mathbb{R}) .
$$

The collection of all such functions will be denoted by $A P(\mathbb{R}, X)$.

This definition is equivalent to the so-called Bochner criterion, namely, $f \in A P(\mathbb{R}, X)$ if and only if for every sequence of reals $\left(s_{n}^{\prime}\right)$ there exists a subsequence $\left(s_{n}\right)$ such that $\left(f\left(\cdot+s_{n}\right)\right)$ is uniformly convergent on $\mathbb{R}$.

Definition 2.2 ([21]) A function $f \in C(\mathbb{R} \times Y, X)$ is called (Bohr) almost periodic in $t \in \mathbb{R}$ uniformly in $y \in K$ where $K \subset Y$ is any compact subset if for each $\epsilon>0$ there exists $l=$ $l(\epsilon)>0$ such that every interval of length $l$ contains a number $\tau$ with the property that

$$
\|f(t+\tau, y)-f(t, y)\|<\epsilon \quad(t \in \mathbb{R}, y \in K) .
$$

The collection of such functions will be denoted by $A P(\mathbb{R} \times Y, X)$.

Definition 2.3 ([22]) A function $f \in C(\mathbb{R}, X)$ is called almost automorphic in $t \in \mathbb{R}$ if for each sequence $\left(s_{n}^{\prime}\right)$ there exists a subsequence $\left(s_{n}\right) \subset\left(s_{n}^{\prime}\right)$ such that

$$
\lim _{n \rightarrow \infty} f\left(t+s_{n}\right)=: \bar{f}(t) \quad \text { and } \quad \lim _{n \rightarrow \infty} \bar{f}\left(t-s_{n}\right)=f(t) \quad \forall t \in \mathbb{R} .
$$

The collection of such functions will be denoted by $A A(\mathbb{R}, X)$.

Definition 2.4 ([22]) A function $f \in C(\mathbb{R} \times X, X)$ is called almost automorphic if $f(t, u)$ is almost automorphic for each $t \in \mathbb{R}$ and for all $u \in B$, where $B$ is any bounded set of $X$. The collection of such functions will be denoted by $A A(\mathbb{R} \times X, X)$. 
Let $U$ denote the set of all functions $\rho: \mathbb{R} \rightarrow(0, \infty)$ in $L_{\text {loc }}^{1}(\mathbb{R})$ such that $\rho(t)>0$ for all $t \in \mathbb{R}$ a.e. For a given $r>0$ and for each $\rho \in U$, we set

$$
m(r, \rho):=\int_{-r}^{r} \rho(t) d t
$$

Thus the space of weights $U_{\infty}$ is defined by

$$
U_{\infty}:=\left\{\rho \in U: \lim _{r \rightarrow \infty} m(r, \rho)=\infty\right\} .
$$

Now, for $\rho \in U_{\infty}$, we define

$$
\begin{aligned}
P A A_{0}(\mathbb{R}, X):=\left\{f \in B C(\mathbb{R}, X): \lim _{r \rightarrow \infty} \frac{1}{m(r, \rho)} \int_{-r}^{r}\|f(t)\| \rho(t) d t=0\right\} ; \\
P A A_{0}(\mathbb{R} \times Y, X):=\{f \in B C(\mathbb{R} \times Y, X): f(\cdot, y) \text { is bounded for each } y \in Y \\
\text { and } \left.\lim _{r \rightarrow \infty} \frac{1}{m(r, \rho)} \int_{-r}^{r}\|f(t)\| \rho(t) d t=0 \text {, uniformly in } y \in Y\right\} .
\end{aligned}
$$

Definition 2.5 ([4] $)$ Let $\rho \in U_{\infty}$. A function $f \in B C(\mathbb{R}, X)$ (respectively $f \in B C(\mathbb{R} \times Y, X)$ ) is called weighted pseudo almost periodic if it can be expressed as $f=g+h$ where $g \in$ $A P(\mathbb{R}, X)$ (respectively $A A(\mathbb{R} \times Y, X))$ and $h \in P A A_{0}(\mathbb{R}, X)$ (respectively $P A A_{0}(\mathbb{R} \times Y, X)$ ). We denote by $\operatorname{WPAP}(\mathbb{R}, X)$ (respectively $\operatorname{WPAP}(\mathbb{R} \times Y, X)$ ) the set of all such functions.

Definition 2.6 ([23]) Let $\rho \in U_{\infty}$. A function $f \in B C(\mathbb{R}, X)$ (respectively $f \in B C(\mathbb{R} \times$ $Y, X)$ ) is called weighted pseudo almost automorphic if it can be expressed as $f=g+$ $h$ where $g \in A A(\mathbb{R}, X)$ (respectively $A A(\mathbb{R} \times Y, X)$ ) and $h \in P A A_{0}(\mathbb{R}, X)$ (respectively $P A A_{0}(\mathbb{R} \times Y, X)$ ). We denote by $W P A A(\mathbb{R}, X)$ (respectively $W P A A(\mathbb{R} \times Y, X)$ ) the set of all such functions.

Definition 2.7 ([1]) The Bochner transform $f^{b}(t, s)$ with $t \in \mathbb{R}, s \in[0,1]$ of a function $f: \mathbb{R} \rightarrow X$ is defined by

$$
f^{b}(t, s):=f(t+s)
$$

Definition 2.8 ([1]) The Bochner transform $f^{b}(t, s, u)$ with $t \in \mathbb{R}, s \in[0,1], u \in X$ of a function $f: \mathbb{R} \times X \rightarrow X$ is defined by

$$
f^{b}(t, s, u):=f(t+s, u) \quad \text { for all } u \in X .
$$

Definition 2.9 ([1]) Let $p \in[1, \infty)$. The space $B S^{p}(\mathbb{R}, X)$ of all Stepanov bounded functions, with exponent $p$, consists of all measurable functions $f: \mathbb{R} \rightarrow X$ such that $f^{b} \in$ $L^{\infty}\left(\mathbb{R}, L^{p}(0,1 ; X)\right)$. This is a Banach space with the norm

$$
\|f\|_{S^{p}}:=\left\|f^{b}\right\|_{L^{\infty}\left(\mathbb{R}, L^{p}\right)}=\sup _{t \in \mathbb{R}}\left(\int_{t}^{t+1}\|f(\tau)\|^{p} d \tau\right)^{\frac{1}{p}} .
$$

Definition 2.10 ([24]) A function $f \in B S^{p}(\mathbb{R}, X)$ is called Stepanov almost periodic if $f^{b} \in$ $A P\left(\mathbb{R}, L^{p}(0,1 ; X)\right)$. We denote the set of all functions by $A P S^{p}(\mathbb{R}, X)$. 
Definition 2.11 ([24]) A function $f: \mathbb{R} \times X \rightarrow Y$ with $f(\cdot, u) \in B S^{p}(\mathbb{R}, Y)$, for each $u \in X$, is called Stepanov almost periodic function in $t \in \mathbb{R}$ uniformly for $u \in X$ if, for each $\epsilon>0$ and each compact set $K \subset X$ there exists a relatively dense set $P=P(\epsilon, f, K) \subset \mathbb{R}$ such that

$$
\sup _{t \in \mathbb{R}}\left(\int_{0}^{1}\|f(t+s+\tau, u)-f(t+s, u)\| d s\right)^{1 / p}<\epsilon
$$

for each $\tau \in P$ and each $u \in K$. We denote by $\operatorname{APS}^{p}(\mathbb{R} \times X, Y)$ the set of such functions.

Definition $2.12([25,26])$ The space $A A S^{p}(\mathbb{R}, X)$ of Stepanov-like automorphic (or $S^{p}$ almost automorphic) functions consists of all $f \in B S^{p}(\mathbb{R}, X)$ such that $f^{b} \in A A\left(\mathbb{R}, L^{p}(0\right.$, $1 ; X)$ ).

Definition 2.13 ([25, 26]) A function $f: \mathbb{R} \times Y \rightarrow X$ given by $(t, u) \mapsto f(t, u)$ with $f(\cdot, u) \in$ $L_{\mathrm{loc}}^{p}(\mathbb{R}, \mathbb{X})$ for each $u \in Y$, is said to be $S^{p}$-almost automorphic in $t \in \mathbb{R}$ uniformly in $u \in Y$ if $t \mapsto f(t, u)$ is $S^{p}$-almost automorphic for each $u \in Y$. This means that for every sequence of real numbers $\left(s_{n}^{\prime}\right)_{n \in \mathbb{N}}$ there exist a subsequence $\left(s_{n}\right)_{n \in \mathbb{N}}$ and a function $g(\cdot, u) \in L_{\text {loc }}^{p}(\mathbb{R}, \mathbb{X})$ such that

$$
\lim _{n \rightarrow \infty}\left(\int_{t}^{t+1}\left\|f\left(s+s_{n}, u\right)-g(s, u)\right\|^{p} d s\right)^{1 / p}=0
$$

and

$$
\lim _{n \rightarrow \infty}\left(\int_{t}^{t+1}\left\|g\left(s-s_{n}, u\right)-f(s, u)\right\|^{p} d s\right)^{1 / p}=0
$$

pointwise on $\mathbb{R}$ and for each $u \in Y$. We denote by $A A S^{p}(\mathbb{R} \times Y, X)$ the set of all such functions.

Definition 2.14 ([1]) Let $\rho \in U_{\infty}$. A function $f \in B S^{p}(\mathbb{R}, X)$ is called Stepanov-like weighted pseudo almost periodic if it can expressed as $f=h+\varphi$ where $h^{b} \in A P\left(\mathbb{R}, L^{p}(0\right.$, $1 ; X))$ and $\varphi^{b} \in P A A_{0}\left(\mathbb{R}, L^{p}(0,1 ; X)\right)$. We denote the set of all functions by $\operatorname{WPAPS}^{p}(\mathbb{R}, X)$.

Definition 2.15 ([1]) Let $\rho \in U_{\infty}$. A function $f: \mathbb{R} \times Y \rightarrow X$ with $f(\cdot, u) \in L_{\mathrm{loc}}^{p}(\mathbb{R}, X)$ for each $u \in X$ is called Stepanov-like weighted pseudo almost periodic if there exist two functions $h, \phi: \mathbb{R} \times Y \rightarrow X$ such that $f=g+\phi$, where $h^{b} \in A P\left(\mathbb{R} \times L^{p}(0,1), X\right)$ and $\phi^{b} \in P A A_{0}\left(\mathbb{R} \times L^{p}(0,1), X\right)$.

Definition 2.16 ([23]) Let $\rho \in U_{\infty}$. A function $f \in B S^{p}(\mathbb{R}, X)$ is called Stepanov-like weighted pseudo almost automorphic if it can expressed as $f=h+\varphi$ where $h^{b} \in$ $A A\left(\mathbb{R}, L^{p}(0,1 ; X)\right)$ and $\varphi^{b} \in P A A_{0}\left(\mathbb{R}, L^{p}(0,1 ; X)\right)$. We denote the set of all functions by $\operatorname{WPAAS}^{p}(\mathbb{R}, X)$.

Definition 2.17 ([23]) Let $\rho \in U_{\infty}$. A function $f: \mathbb{R} \times Y \rightarrow X$ with $f(\cdot, u) \in L_{\mathrm{loc}}^{p}(\mathbb{R}, X)$ for each $u \in X$ is called Stepanov-like weighted pseudo almost periodic if there exist two functions $h, \phi: \mathbb{R} \times Y \rightarrow X$ such that $f=g+\phi$, where $h^{b} \in A P\left(\mathbb{R} \times L^{p}(0,1), X\right)$ and $\phi^{b} \in$ $P A A_{0}\left(\mathbb{R} \times L^{p}(0,1), X\right)$. 
Now, we introduce a (natural) linear operator from $B S^{p}(\mathbb{R}, X)$ into $L^{\infty}\left(\mathbb{R}, L^{p}(0,1 ; X)\right)$, which will be an important tool in order to clarify some concepts and achieve our goals.

Definition 2.18 We define

$$
\begin{aligned}
& \mathcal{B}: B S^{p}(\mathbb{R}, X) \rightarrow L^{\infty}\left(\mathbb{R}, L^{p}(0,1 ; X)\right), \\
& f \mapsto(\mathcal{B} f)(t)(s)=f(t+s) .
\end{aligned}
$$

Remark 2.19 It follows from the definition that the operator $\mathcal{B}$ is a linear isometry between $B S^{p}(\mathbb{R}, X)$ and $L^{\infty}\left(\mathbb{R}, L^{p}(0,1 ; X)\right)$. More precisely

$$
\|\mathcal{B} f\|_{\infty}=\|f\|_{B S^{p}(\mathbb{R}, X)}
$$

Remark 2.20 The definition of Stepanov-like weighted pseudo almost periodic functions given by Diagana in [1] can be written using the preceding notation. Thus, for $\rho \in U_{\infty}$, we say that a function $f$ is Stepanov-like weighted pseudo almost periodic (or $S^{p}$-weighted pseudo almost periodic) if and only if $f \in \mathcal{B}^{-1}\left(A P\left(\mathbb{R}, L^{p}(0,1 ; X)\right)\right)+$ $\mathcal{B}^{-1}\left(P A A_{0}\left(\mathbb{R}, L^{p}(0,1 ; X)\right)\right)$. Then

$$
\operatorname{WPAPS}^{p}(\mathbb{R}, X)=\mathcal{B}^{-1}\left(A P\left(\mathbb{R}, L^{p}(0,1 ; X)\right)\right)+\mathcal{B}^{-1}\left(P A A_{0}\left(\mathbb{R}, L^{p}(0,1 ; X)\right)\right) .
$$

Moreover, since $\mathcal{B}$ is an isometry and $A P\left(\mathbb{R}, L^{p}(0,1 ; X)\right) \cap P A A_{0}\left(\mathbb{R}, L^{p}(0,1 ; X)\right)=\{0\}$ then the sum is direct, that is,

$$
\operatorname{WPAPS}^{p}(\mathbb{R}, X)=\mathcal{B}^{-1}\left(A P\left(\mathbb{R}, L^{p}(0,1 ; X)\right)\right) \oplus \mathcal{B}^{-1}\left(P A A_{0}\left(\mathbb{R}, L^{p}(0,1 ; X)\right)\right) .
$$

Based on the definition of the operator $\mathcal{B}$, we prove that the $\operatorname{WPAPS}^{p}(\mathbb{R}, X)$ is a Banach space when endowed with their natural norm.

Theorem 2.21 $\operatorname{WPAPS}^{p}(\mathbb{R}, X)$ is a Banach space with the norm

$$
\|f\|_{\operatorname{WPAPS}_{(\mathbb{R}, X)}}=\|g\|_{B S^{p}(\mathbb{R}, X)}+\|h\|_{B S^{p}(\mathbb{R}, X)}
$$

where $f=g+h$ with $g \in \mathcal{B}^{-1}\left(A P\left(\mathbb{R}, L^{p}(0,1 ; X)\right)\right)$ and $h \in \mathcal{B}^{-1}\left(P A A_{0}\left(\mathbb{R}, L^{p}(0,1 ; X)\right)\right)$.

Proof Let $\left(f_{n}\right)$ be a Cauchy sequence in $\operatorname{WPAPS}^{p}(\mathbb{R}, X)$. Then $\left\|f_{n}-f_{m}\right\|_{\operatorname{WPAPS}_{(\mathbb{R}, X)} \rightarrow 0}$ if $n, m \rightarrow \infty$. Let $f_{n}=g_{n}+h_{n}$ and $f_{m}=g_{m}+h_{m}$ with $g_{n}, g_{m} \in \mathcal{B}^{-1}\left(A P\left(\mathbb{R}, L^{p}(0,1 ; X)\right)\right)$ and $h_{n}, h_{m} \in \mathcal{B}^{-1}\left(P A A_{0}\left(\mathbb{R}, L^{p}(0,1 ; X)\right)\right)$. If $n, m \rightarrow \infty$, then

$$
\left\|\mathcal{B} g_{n}-\mathcal{B} g_{m}\right\|_{L^{\infty}\left(\mathbb{R}, L^{p}\right)}=\left\|g_{n}-g_{m}\right\|_{B S^{p}(\mathbb{R}, X)} \leq\left\|f_{n}-f_{m}\right\|_{W P A P S^{p}(\mathbb{R}, X)} \rightarrow 0
$$

and

$$
\left\|\mathcal{B} g_{n}-\mathcal{B} g_{m}\right\|_{L^{\infty}\left(\mathbb{R}, L^{p}\right)}=\left\|g_{n}-g_{m}\right\|_{B S^{p}(\mathbb{R}, X)} \leq\left\|f_{n}-f_{m}\right\|_{\operatorname{WPAPS}^{p}(\mathbb{R}, X)} \rightarrow 0
$$

This implies that $\left(\mathcal{B} g_{n}\right)$ and $\left(\mathcal{B} h_{n}\right)$ are Cauchy sequences in $A P\left(\mathbb{R}, L^{p}(0,1 ; X)\right)$ and $P A A_{0}(\mathbb{R}$, $\left.L^{p}(0,1 ; X)\right)$, respectively. Since $A P\left(\mathbb{R}, L^{p}(0,1 ; X)\right)$ and $P A A_{0}\left(\mathbb{R}, L^{p}(0,1 ; X)\right)$ are Banach 
spaces (see [21] and [27], respectively) then there exist $g \in A P\left(\mathbb{R}, L^{p}(0,1 ; X)\right)$ and $h \in$ $P A A_{0}\left(\mathbb{R}, L^{p}(0,1 ; X)\right)$ such that

$$
\left\|\mathcal{B} g_{n}-g\right\|_{L^{\infty}\left(\mathbb{R}, L^{p}\right)} \rightarrow 0, \quad\left\|\mathcal{B} h_{n}-h\right\|_{L^{\infty}\left(\mathbb{R}, L^{p}\right)} \rightarrow 0 \quad(n \rightarrow \infty)
$$

Let $f_{1}:=\mathcal{B}^{-1}(\{g\}) \in \mathcal{B}^{-1}\left(A P\left(\mathbb{R}, L^{p}(0,1 ; X)\right)\right)$ and $f_{2}:=\mathcal{B}^{-1}(\{h\}) \in \mathcal{B}^{-1}\left(P A A_{0}\left(\mathbb{R}, L^{p}(0,1 ; X)\right)\right)$. Note that $f_{1}$ and $f_{2}$ are well defined because $\mathcal{B}$ is injective. Let $f:=f_{1}+f_{2} \in \operatorname{WPAPS}^{p}(\mathbb{R}, X)$.

Then

$$
\begin{aligned}
\left\|f_{n}-f\right\|_{\operatorname{WPAPS}(\mathbb{R}, X)} & =\left\|\left(g_{n}+h_{n}\right)-\left(f_{1}+f_{2}\right)\right\|_{\operatorname{WPAPS}_{(\mathbb{R}, X)}} \\
& =\left\|g_{n}-f_{1}\right\|_{B S^{p}(\mathbb{R}, X)}+\left\|h_{n}-f_{2}\right\|_{B S^{p}(\mathbb{R}, X)} \\
& =\left\|\mathcal{B} g_{n}-\mathcal{B} f_{1}\right\|_{L^{\infty}\left(\mathbb{R}, L^{p}\right)}+\left\|\mathcal{B} h_{n}-\mathcal{B} f_{2}\right\|_{L^{\infty}\left(\mathbb{R}, L^{p}\right)} \\
& =\left\|\mathcal{B} g_{n}-g\right\|_{L^{\infty}\left(\mathbb{R}, L^{p}\right)}+\left\|\mathcal{B} h_{n}-h\right\|_{L^{\infty}\left(\mathbb{R}, L^{p}\right)} \rightarrow 0 \quad(n \rightarrow \infty) .
\end{aligned}
$$

Therefore $\operatorname{WPAPS}^{p}(\mathbb{R}, X)$ is a Banach space.

Remark 2.22 Similarly, we can equivalently define the space of Stepanov-like weighted pseudo almost automorphic functions as

$$
\operatorname{WPAAS}^{p}(\mathbb{R}, X)=\mathcal{B}^{-1}\left(A A\left(\mathbb{R}, L^{p}(0,1 ; X)\right)\right) \oplus \mathcal{B}^{-1}\left(P A A_{0}\left(\mathbb{R}, L^{p}(0,1 ; X)\right)\right) .
$$

Following the same idea as above, we can prove that $\operatorname{WPAAS}^{p}(\mathbb{R}, X)$ is a Banach space with the norm

$$
\|f\|_{\operatorname{WPAAS}^{p}(\mathbb{R}, X)}=\|g\|_{B S^{p}(\mathbb{R}, X)}+\|h\|_{B S^{p}(\mathbb{R}, X)},
$$

where $f=g+h$ with $g \in \mathcal{B}^{-1}\left(A A\left(\mathbb{R}, L^{p}(0,1 ; X)\right)\right)$ and $h \in \mathcal{B}^{-1}\left(P A A_{0}\left(\mathbb{R}, L^{p}(0,1 ; X)\right)\right)$.

\section{Convolution theorems}

In this section, we establish convolution theorems for Stepanov-like almost periodic and Stepanov-like weighted almost periodic functions. The relevant fact is that the finite convolution includes a general strongly continuous family of bounded and linear operators instead of only $C_{0}$-semigroups.

Theorem 3.1 Let $S: \mathbb{R} \rightarrow B(X)$ be strongly continuous. Suppose that there exists a function $\phi \in L^{1}(\mathbb{R})$ such that:

(a) $\|S(t)\| \leq \phi(t), t \in \mathbb{R}$;

(b) $\phi(t)$ is increasing;

(c) $\sum_{n=1}^{\infty} \phi(n)<\infty$.

If $g \in \operatorname{APS}^{p}(\mathbb{R}, X)$, then

$$
(S * g)(t):=\int_{-\infty}^{t} S(t-s) g(s) d s \in A P(\mathbb{R}, X) .
$$

Proof Let $g \in A P S^{p}(\mathbb{R}, X)=\mathcal{B}^{-1}\left(A P\left(\mathbb{R}, L^{p}(0,1 ; X)\right)\right)$ and

$$
H_{k}(t):=\int_{t-k}^{t-k+1} S(t-s) g(s) d s=\int_{k-1}^{k} S(s) g(t-s) d s .
$$


We show that $H_{k}(t)$ is continuous. Indeed

$$
\begin{aligned}
\left\|H_{k}(t+h)-H_{k}(t)\right\| & \leq \int_{k-1}^{k}\|S(s)\|\|g(t+h-s)-g(t-s)\| d s \\
& \leq \int_{k-1}^{k} \phi(s)\|g(t+h-s)-g(t-s)\| d s \\
& =\phi(k-1) \int_{t-k}^{t-k+1}\|g(t+h-s)-g(t-s)\| d s \rightarrow 0 \quad(h \rightarrow 0) .
\end{aligned}
$$

We claim that $H_{k}(t) \in A P(\mathbb{R}, X)$ for each $k \in \mathbb{N}$. Since $g \in \mathcal{B}^{-1}\left(A P\left(\mathbb{R}, L^{p}(0,1 ; X)\right)\right), \mathcal{B} g \in$ $A P\left(\mathbb{R}, L^{p}(0,1 ; X)\right)$. Hence, for all sequences of real numbers $\left(s_{n}^{\prime}\right)$ there exist a subsequence $\left(s_{n}\right)$ of $\left(s_{n}^{\prime}\right)$ and a function $h$ such that

$$
\left\|(\mathcal{B} g)\left(t+s_{n}\right)-h(t)\right\|_{p} \rightarrow 0 \quad(n \rightarrow \infty)
$$

uniformly on $t \in \mathbb{R}$. Let $H(t):=\int_{k-1}^{k} S(s) h(t-s) d s$. Then for each $k \in \mathbb{N}$ we have

$$
\begin{aligned}
\left\|H_{k}\left(t+s_{n}\right)-H(t)\right\| & =\left\|\int_{k-1}^{k} S(s) g\left(t+s_{n}-s\right) d s-\int_{k-1}^{k} S(s) h(t-s) d s\right\| \\
& \leq \int_{k-1}^{k}\left\|g\left(t+s_{n}-s\right)-h(t-s)\right\| d s \\
& =\phi(0) \int_{0}^{1}\left\|g\left(t+s_{n}-k+p\right)-h(t-k+p)\right\| d p \\
& \leq \phi(0)\left\|(\mathcal{B} g)\left(t+s_{n}-k\right)-h(t-k)\right\|_{p} \rightarrow 0 \quad(n \rightarrow \infty)
\end{aligned}
$$

uniformly on $t \in \mathbb{R}$. Therefore $H_{k}(t) \in A P(\mathbb{R}, X)$. Now

$$
\begin{aligned}
\left\|H_{k}(t)\right\| & \leq \int_{t-k}^{t-k+1}\|S(t-s)\|\|g(s)\| d s \\
& =\int_{k-1}^{k}\|S(s)\|\|g(t-s)\| d s \\
& \leq \int_{k-1}^{k} \phi(s)\|g(t-s)\| d s \\
& \leq \phi(k-1)\left(\int_{k-1}^{k}\|g(t-s)\|^{p} d s\right)^{1 / p} .
\end{aligned}
$$

The last estimation implies that

$$
\left\|H_{k}(t)\right\| \leq \phi(k-1)\|g\|_{B S^{p}}
$$

By hypothesis we have

$$
\sum_{k=1}^{\infty}\left\|H_{k}(t)\right\| \leq \sum_{k=1}^{\infty} \phi(k-1)\|g\|_{B S^{p}}<C\|g\|_{B S^{p}}<\infty .
$$


It follows from the Weierstrass test that the series $\sum_{k=1}^{\infty} H_{k}(t)$ is uniformly convergent on $\mathbb{R}$. Moreover,

$$
H(t)=\int_{-\infty}^{t} S(t-s) h(s) d s=\sum_{k=1}^{\infty} H_{k}(t)
$$

Hence $(S * g)(t):=\int_{-\infty}^{t} S(t-s) g(s) d s \in A P(\mathbb{R}, X)$.

Remark 3.2 Using similar arguments we can prove that if $g \in A A S^{p}(\mathbb{R}, X)$ then $(S * f)(t):=$ $\int_{-\infty}^{t} S(t-s) f(s) d s \in A A(\mathbb{R}, X)$.

The following is the main result in this section.

Theorem 3.3 Let $\rho \in U_{\infty}$ be given and let $S: \mathbb{R} \rightarrow B(X)$ be strongly continuous. Suppose that there exists a function $\phi \in L^{1}(\mathbb{R})$ such that:

(a) $\|S(t)\| \leq \phi(t), t \in \mathbb{R}$;

(b) $\phi(t)$ is increasing;

(c) $\sum_{n=1}^{\infty} \phi(n)<\infty$.

Suppose that $f=g+h \in \operatorname{WPAPS}^{p}(\mathbb{R}, X)$ with $g \in \mathcal{B}^{-1}\left(A P\left(\mathbb{R}, L^{p}(0,1 ; X)\right)\right)$ and $h \in$ $\mathcal{B}^{-1}\left(P A A_{0}\left(\mathbb{R}, L^{p}(0,1 ; X)\right)\right)$. Then

$$
(S * f)(t):=\int_{-\infty}^{t} S(t-s) f(s) d s \in W P A P(\mathbb{R}, X) .
$$

Proof We have

$$
(S * f)(t):=\int_{-\infty}^{t} S(t-s) f(s) d s=\int_{-\infty}^{t} S(t-s) g(s) d s+\int_{-\infty}^{t} S(t-s) h(s) d s .
$$

It follows from Theorem 3.1 that $(S * g)(t) \in A P(\mathbb{R}, X)$. It remains to show that $(S * h)(t) \in$ $P A A_{0}(\mathbb{R}, X)$. Set

$$
H(t):=\int_{-\infty}^{t} S(t-s) h(s) d s=\int_{-\infty}^{t} S(s) h(t-s) d s
$$

and define

$$
H_{n}(t):=\int_{t-n}^{t-n+1} S(t-\sigma) h(\sigma) d \sigma, \quad n=1,2, \ldots
$$

As above, we can prove that $H_{n}(t)$ is continuous. Then

$$
\begin{aligned}
\left\|H_{n}(t)\right\| & \leq \int_{t-n}^{t-n+1}\|S(t-\sigma)\|\|h(\sigma)\| d \sigma \\
& =\int_{n-1}^{n}\|S(\sigma)\|\|h(t-\sigma)\| d \sigma \\
& \leq \int_{n-1}^{n} \phi(s)\|h(t-\sigma)\| d \sigma \\
& \leq \phi(n-1)\left(\int_{n-1}^{n}\|h(t-\sigma)\|^{p} d \sigma\right)^{1 / p} .
\end{aligned}
$$


Then for $r>0$,

$$
\begin{aligned}
& \frac{1}{m(r, \rho)} \int_{-r}^{r}\left\|H_{n}(t)\right\| \rho(t) d t \\
& \quad \leq \phi(n-1) \frac{1}{m(r, \rho)} \int_{-r}^{r}\left(\int_{n-1}^{n}\|h(t-\sigma)\|^{p} d \sigma\right)^{1 / p} \rho(t) d t .
\end{aligned}
$$

Using the fact that the space $P A A_{0}(\mathbb{R}, X)$ is translation invariant, it follows that $t \rightarrow h(t-\sigma)$ belongs to $P A A_{0}(\mathbb{R}, X)$. The above inequality leads to $H_{n}(t) \in P A A_{0}(\mathbb{R}, X)$ for each $n=$ $1,2, \ldots$ The last estimation implies that

$$
\left\|H_{n}(t)\right\| \leq \phi(n-1)\|h\|_{S^{p}} .
$$

By hypothesis we have

$$
\sum_{n=1}^{\infty}\left\|H_{n}(t)\right\| \leq \sum_{n=1}^{\infty} \phi(n-1)\|h\|_{B S^{p}}<C\|h\|_{B S^{p}}<\infty .
$$

It follows from the Weierstrass test that the series $\sum_{n=1}^{\infty} H_{n}(t)$ is uniformly convergent on $\mathbb{R}$. Moreover,

$$
H(t)=\int_{-\infty}^{t} S(t-s) h(s) d s=\sum_{n=1}^{\infty} H_{n}(t)
$$

We note that $H(t) \in C(\mathbb{R}, X)$ and

$$
\|H(t)\| \leq \sum_{n=1}^{\infty}\left\|H_{n}(t)\right\| \leq C\|h\|_{S^{p}}
$$

Now,

$$
\begin{aligned}
\frac{1}{m(r, \rho)} \int_{-r}^{r}\|H(t)\| \rho(t) d t \leq & \frac{1}{m(r, \rho)} \int_{-r}^{r}\left\|H(t)-\sum_{k=1}^{n} H_{k}(t)\right\| \rho(t) d t \\
& +\sum_{k=1}^{n} \frac{1}{m(r, \rho)} \int_{-r}^{r}\left\|H_{k}(t)\right\| \rho(t) d t .
\end{aligned}
$$

Since $H_{k}(t) \in P A A_{0}(\mathbb{R}, X)$ and $\sum_{k=1}^{n} H_{n}(t)$ converges uniformly to $H(t)$, we have

$$
\lim _{r \rightarrow \infty} \frac{1}{m(r, \rho)} \int_{-r}^{r}\|H(t)\| \rho(t) d t=0 .
$$

It follows that $H(\cdot)=\sum_{n=1}^{\infty} H_{n}(t) \in P A A_{0}(\mathbb{R}, X)$. Therefore, $(S * f)(t):=\int_{-\infty}^{t} S(t-s) f(s) d s$ is weighted pseudo almost periodic.

Remark 3.4 Using similar arguments, we can prove that if $f \in W P A A S^{p}(\mathbb{R}, X)$ then $(S *$ $f)(t):=\int_{-\infty}^{t} S(t-s) f(s) d s \in W P A A(\mathbb{R}, X)$. 


\section{Weighted pseudo almost periodic mild solutions}

In this section we consider the problem of existence and uniqueness of weighted pseudo almost periodic mild solutions for the following class of integro-differential equations:

$$
u^{\prime}(t)=A u(t)+\alpha \int_{-\infty}^{t} e^{-\beta(t-s)} A u(s) d s+f(t, u(t)) .
$$

Note that (4.1) has the form of (1.1) with $L u=u^{\prime}(t)-A u(t)-\alpha \int_{-\infty}^{t} e^{-\beta(t-s)} A u(s) d s$.

Now, we recall some results of uniform exponential stability of solutions to the homogeneous abstract Volterra equation

$$
u^{\prime}(t)=A u(t)+\alpha \int_{-\infty}^{t} e^{-\beta(t-s)} A u(s) d s
$$

Definition 4.1 We say that a solution of (4.2) is uniformly exponentially bounded if for some $\omega \in \mathbb{R}$ there exists a constant $M>0$ such that

$$
\|u(t)\| \leq M e^{-\omega t} \quad(t \geq 0) .
$$

In particular, we say that the solution is uniformly stable if $\omega>0$.

Definition 4.2 Let $X$ be a Banach space. A strongly continuous function $S: \mathbb{R}_{+} \rightarrow B(X)$ is said to be immediately norm continuous if $S:(0, \infty) \rightarrow B(X)$ is continuous.

We recall the following remarkable result from [28].

Theorem 4.3 Let $\beta>0, \alpha \neq 0$, and $\alpha+\beta>0$ be given. Assume that:

(a) A generates an immediately norm continuous $C_{0}$-semigroup on a Banach space $X$;

(b) $\sup \left\{\Re \lambda, \lambda \in \mathbb{C}: \lambda(\lambda+\beta)(\lambda+\alpha+\beta)^{-1} \in \sigma(A)\right\}<0$.

Then the solutions of problem (4.2) are uniformly exponentially stable.

We note in the next proposition that under the given hypothesis on $A$, it is possible to construct a strongly continuous family of bounded and linear operators for (4.2) that commutes with $A$ and satisfies a certain 'resolvent equation'. This class of strongly continuous families has been studied extensively in the literature of abstract Volterra equations; see e.g. Prüss [3] and references therein.

Proposition 4.4 ([2]) Let $\beta>0, \alpha \neq 0$, and $\alpha+\beta>0$. Assume that:

(i) A generates an immediately continuous $C_{0}$-semigroup on a Banach space $X$;

(ii) $\sup \left\{\Re \lambda, \lambda \in \mathbb{C}: \lambda(\lambda+\beta)(\lambda+\alpha+\beta)^{-1} \in \sigma(A)\right\}<0$.

Then there exists a uniformly exponentially stable and strongly continuous family of operators $(S(t))_{t \geq 0}$ such that

(a) $S(t) D(A) \subset D(A)$;

(b) $S(t) A x=A S(t) x \forall x \in D(A), \forall t \geq 0$;

(c)

$$
S(t) x=x+\int_{0}^{t} b(t-s) A S(s) x d s \quad \forall x \in X, \forall t \geq 0,
$$

where $b(t)=1+\frac{\alpha}{\beta}\left(1-e^{-\beta t}\right), t \geq 0$. 
According to the above proposition, there exist constants $M>0$ and $\omega>0$ such that

$$
\|S(t)\| \leq M e^{-\omega t}, \quad t \geq 0
$$

In particular, we can state the following definition.

Definition 4.5 Let $f: \mathbb{R} \times X \rightarrow X$ be given. A continuous function $u: \mathbb{R} \rightarrow X$ is said to be a mild solution of (4.1) if it satisfies

$$
u(t)=\int_{-\infty}^{t} S(t-s) f(s, u(s)) d s \quad(t \in \mathbb{R})
$$

where $(S(t))_{t \geq 0}$ is given in Proposition 4.4.

We recall the following composition theorem.

Theorem 4.6 ([1]) Let $\rho \in U_{\infty}$ and let $f: \mathbb{R} \times X \rightarrow Y$ be a $S^{p}$-weighted pseudo almost periodic function. Suppose that $f(t, u)$ is Lipschitzian in $u \in X$ uniformly in $t \in \mathbb{R}$, that is, there exists $L_{f}>0$ such that

$$
\|f(t, u)-f(t, v)\|_{Y} \leq L_{f}\|u-v\|_{X}
$$

for all $t \in \mathbb{R}$ and for all $u, v \in X$. If $\phi \in W_{P A P S}(\mathbb{R}, X)$ then the function $\Gamma: \mathbb{R} \rightarrow X$ defined by $\Gamma(\cdot)=f(\cdot, \phi(\cdot))$ belongs to $\operatorname{WPAP}(\mathbb{R}, X)$.

In [2] the authors obtained the existence and uniqueness of mild solutions for the semilinear equations with infinite delay on a class of spaces which do not include Stepanov type functions as the forcing term. Next, we can obtain the existence and uniqueness of weighted pseudo almost periodic solutions with the help of Theorem 3.3 and Theorem 4.6.

Theorem 4.7 Let $\rho \in U_{\infty}, \beta>0, \alpha \neq 0$, and $\alpha+\beta>0$. Suppose that:

(H1) A generates an immediately norm continuous semigroup on a Banach space $X$ and $\sup \left\{\Re \lambda, \lambda \in \mathbb{C}: \lambda(\lambda+\beta)(\lambda+\alpha+\beta)^{-1} \in \sigma(A)\right\}<0$.

(H2) $f \in \operatorname{WPAPS}^{p}(\mathbb{R} \times X, X)$ and there exists a constant $L_{f}>0$ such that

$$
\|f(t, u)-f(t, v)\| \leq L_{f}\|u-v\|, \quad t \in \mathbb{R}, u, v \in X .
$$

(H3) $\frac{M L_{f}}{\omega}<1$, where $M>0$ and $\omega>0$ are given in (4.4).

Then (4.1) has a unique mild solution in $\operatorname{WPAP}(\mathbb{R}, X)$.

Proof By Proposition 4.4, hypothesis (H1) implies that there exists a strongly continuous family $(S(t))_{t \geq 0}$ such that $\|S(t)\| \leq M e^{-\omega t}$ for some $M, \omega>0$. Consider the operator $Q$ : $\operatorname{WPAP}(\mathbb{R}, X) \rightarrow \operatorname{WPAP}(\mathbb{R}, X)$ defined by

$$
Q(u)(t):=\int_{-\infty}^{t} S(t-s) f(s, u(s)) d s \quad(t \in \mathbb{R}) .
$$

First, we show that $Q(W P A P(\mathbb{R}, X)) \subset W P A P(\mathbb{R}, X)$. Let $u \in W P A P(\mathbb{R}, X)$. Then $u \in$ $\operatorname{WPAPS}^{p}(\mathbb{R}, X)$. It follows from the composition Theorem 4.6 that $f(\cdot, u(\cdot)) \in \operatorname{WPAPS}^{p}(\mathbb{R}$, 
$X)$. On the other hand, the family $(S(t))_{t \geq 0}$ and the function $\phi(t)=M e^{-\omega t}$ satisfy the hypothesis of Theorem 3.3. Therefore $Q(u)(t) \in \operatorname{WPAPS}^{p}(\mathbb{R}, X)$. Now, if $u, v \in \operatorname{WPAP}(\mathbb{R}, X)$ we have

$$
\begin{aligned}
\|Q(u)(t)-Q(v)(t)\|_{\infty} & =\sup _{t \in \mathbb{R}}\left\|\int_{-\infty}^{t} S(t-s)[f(s, u(s))-f(s, v(s))] d s\right\| \\
& \leq L_{f} \sup _{t \in \mathbb{R}} \int_{0}^{\infty}\|S(s)\|_{\infty}\|u(t-s)-v(t-s)\| d s \\
& \leq L_{f}\|u-v\|_{\infty} \int_{0}^{\infty} M e^{-\omega t} d s \\
& =\frac{M L_{f}\|u-v\|_{\infty}}{\omega}<\|u-v\|_{\infty} .
\end{aligned}
$$

This proves that $Q$ is a contraction, so by the Banach fixed point theorem $Q$ has a unique fixed point. It follows that $Q(u)=u \in \operatorname{WPAP}(\mathbb{R}, X)$ is unique. Hence $u$ is the unique mild solution of (4.1).

In what follows, we want to elaborate an alternative existence and uniqueness result with weaker conditions than the Lipschitz continuity imposed on the second variable of the nonlinear forcing term $f$. In order to do that, we first recall the following lemma.

Lemma 4.8 ([29]) Suppose that:

(a) $f \in \operatorname{APS}^{p}(\mathbb{R} \times X, X)$ with $p>1$ and there exist a function $L_{f} \in B S^{r}(\mathbb{R}, \mathbb{R})$

$(r \geq \max \{p, p / p-1\})$ such that

$$
\|f(t, u)-f(t, v)\| \leq L_{f}(t)\|u-v\|, \quad t \in \mathbb{R}, u, v \in X
$$

(b) $x \in A P S^{p}(\mathbb{R}, X)$, and there exists a set $E \subset \mathbb{R}$ with $\operatorname{mes}(E)=0$ such that

$$
K=\overline{\{x(t): t \in \mathbb{R} \backslash E\}}
$$

is compact in $X$. Then there exists $q \in[1, p)$ such that $f(\cdot, x(\cdot)) \in \operatorname{APS}^{q}(\mathbb{R}, X)$.

The next result is a new composition theorem which help us to prove the second main theorem of this paper (see Theorem 4.10 below).

Theorem 4.9 Let $\rho \in U_{\infty}, p>1, f=g+\phi \in W_{P A P S}(\mathbb{R} \times X, X)$ with $g \in \mathcal{B}^{-1}(A P(\mathbb{R} \times$ $\left.\left.X, L^{p}(0,1 ; X)\right)\right)$ and $\phi \in \mathcal{B}^{-1}\left(P A A_{0}\left(\mathbb{R} \times X, L^{p}(0,1 ; X)\right)\right)$. Assume that:

(i) There exist nonnegative functions $L_{f}, L_{g} \in A P S^{p}(\mathbb{R}, \mathbb{R})$ where $r \geq \max \{p, p / p-1\}$ such that

$$
\begin{aligned}
& \|f(t, u)-f(t, v)\| \leq L_{f}(t)\|u-v\|, \\
& \|g(t, u)-g(t, v)\| \leq L_{g}(t)\|u-v\|, \quad t \in \mathbb{R}, u, v \in X .
\end{aligned}
$$

(ii) $h=\alpha+\beta \in \operatorname{WPAPS}^{p}(\mathbb{R}, X)$ with

$$
\alpha \in \mathcal{B}^{-1}\left(A P\left(\mathbb{R}, L^{p}(0,1 ; X)\right)\right), \quad \beta \in \mathcal{B}^{-1}\left(P A A_{0}\left(\mathbb{R}, L^{p}(0,1 ; X)\right)\right)
$$


and there exists a set $E \subset \mathbb{R}$ with $\operatorname{mes}(E)=0$ such that

$$
K=\overline{\{h(t): t \in \mathbb{R} \backslash E\}}
$$

is compact in $X$. Then there exists $q \in[1, p)$ such that $f(\cdot, h(\cdot)) \in \operatorname{WPAPS}^{q}(\mathbb{R}, X)$.

Proof We can decompose

$$
f(t, h(t))=g(t, \alpha(t))+f(t, h(t))-f(t, \alpha(t))+\phi(t, \alpha(t)) .
$$

Set

$$
F(t):=g(t, \alpha(t)), \quad G(t):=f(t, h(t))-f(t, \alpha(t)), \quad H(t):=\phi(t, \alpha(t)) .
$$

Since $r \geq \frac{p}{p-1}$, there exists $q \in[1, p)$ such that $r=\frac{p q}{p-q}$. Let $p^{\prime}=p / p-q$ and $q^{\prime}=p / q$. Therefore $\frac{1}{p^{\prime}}+\frac{1}{q^{\prime}}=1$. Since $\alpha \in A P(\mathbb{R}, X)$ and $g \in A P S^{p}(\mathbb{R} \times X, X)$ then by the assumptions and Lemma 4.8 we obtain $F(t) \in \mathcal{B}^{-1}\left(A P\left(\mathbb{R}, L^{q}(0,1 ; X)\right)\right)$.

Next we show that $G(t) \in \mathcal{B}^{-1}\left(P A A_{0}\left(\mathbb{R}, L^{q}(0,1 ; X)\right)\right)$. By the Hölder inequality we have

$$
\begin{aligned}
\int_{t}^{t+1}\|G(\sigma)\|^{q} d \sigma & =\int_{t}^{t+1}\|f(\sigma, h(\sigma))-f(\sigma, \alpha(\sigma))\|^{q} d \sigma \\
& \leq \int_{t}^{t+1} L_{f}^{q}(\sigma)\|h(\sigma)-\alpha(\sigma)\|^{q} d \sigma \\
& =\int_{t}^{t+1} L_{f}^{q}(\sigma)\|\beta(\sigma)\|^{q} d \sigma \\
& \leq\left(\int_{t}^{t+1} L_{f}^{q p^{\prime}}(\sigma) d \sigma\right)^{1 / p^{\prime}}\left(\int_{t}^{t+1}\|\beta(\sigma)\|^{q q^{\prime}} d \sigma\right)^{1 / q^{\prime}} \\
& =\left[\left(\int_{t}^{t+1} L_{f}^{r}(\sigma) d \sigma\right)^{1 / r}\right]^{r / p^{\prime}}\left[\left(\int_{t}^{t+1}\|\beta(\sigma)\|^{p} d \sigma\right)^{1 / p}\right]^{p / q^{\prime}} \\
& \leq\left\|L_{f}\right\|_{B S^{r}}^{q}\left[\left(\int_{t}^{t+1}\|\beta(\sigma)\|^{p} d \sigma\right)^{1 / p}\right]^{q} .
\end{aligned}
$$

Then

$$
\begin{aligned}
& \frac{1}{m(r, \rho)} \int_{-r}^{r}\left(\int_{t}^{t+1} \rho(t)\|G(\sigma)\|^{q} d \sigma\right)^{1 / q} d t \\
& \leq \frac{\left\|L_{f}\right\|_{B S}}{m(r, \rho)} \int_{-r}^{r} \rho(t)\left(\int_{t}^{t+1}\|\beta(\sigma)\|^{p} d \sigma\right)^{1 / p} d t .
\end{aligned}
$$

Since $\beta(\cdot) \in \mathcal{B}^{-1}\left(P A A_{0}\left(\mathbb{R}, L^{p}(0,1 ; X)\right)\right)$ we obtain $G(\cdot) \in \mathcal{B}^{-1}\left(P A A_{0}\left(\mathbb{R}, L^{q}(0,1 ; X)\right)\right)$.

Next, we prove that $H(\cdot) \in \mathcal{B}^{-1}\left(P A A_{0}\left(\mathbb{R}, L^{q}(0,1 ; X)\right)\right)$. Since $\phi \in \mathcal{B}^{-1}\left(P A A_{0}\left(\mathbb{R} \times X, L^{p}(0\right.\right.$, $1 ; X))$ ), for any $\epsilon>0$ there exists $r_{0}>0$ such that $r>r_{0}$ implies that

$$
\frac{1}{m(r, \rho)} \int_{-r}^{r} \rho(t)\left(\int_{t}^{t+1}\|\phi(\sigma, u)\|^{p} d \sigma\right)^{1 / p} d t<\epsilon \quad(u \in X)
$$


Since $K$ is compact, we can find finite open balls $O_{k}(k=1,2,3, \ldots, m)$ with center $x_{k}$ such that $K \subset \bigcup_{k=1}^{m} O_{k}$. Thus, for all $u \in K$ there exists $x_{k}$ such that

$$
\begin{aligned}
\|\phi(t+\sigma, u)\| \leq & \left\|\phi(t+\sigma, u)-\phi\left(t+\sigma, x_{k}\right)\right\|+\left\|\phi\left(t+\sigma, x_{k}\right)\right\| \\
\leq & \left\|f(t+\sigma, u)-f\left(t+\sigma, x_{k}\right)\right\| \\
& \quad+\left\|g(t+\sigma, u)-g\left(t+\sigma, x_{k}\right)\right\|+\left\|\phi\left(t+\sigma, x_{k}\right)\right\| \\
\leq & L_{f}(t+\sigma) \epsilon+L_{g}(t+\sigma) \epsilon+\left\|\phi\left(t+\sigma, x_{k}\right)\right\| \quad(t \in \mathbb{R}, \sigma \in[0,1]) .
\end{aligned}
$$

Hence

$$
\sup _{u \in K}\|\phi(t+\sigma, u)\| \leq L_{f}(t+\sigma) \epsilon+L_{g}(t+\sigma) \epsilon+\sum_{k=1}^{m}\left\|\phi\left(t+\sigma, x_{k}\right)\right\| .
$$

Since $r \geq p, L_{f}, L_{g} \in A P S^{r}(\mathbb{R}, \mathbb{R}) \subset A P S^{p}(\mathbb{R}, \mathbb{R}) \subset B S^{p}(\mathbb{R}, \mathbb{R})$.

By Minkowskii's inequality, we obtain

$$
\begin{aligned}
& {\left[\int_{0}^{1}\left(\sup _{u \in K}\|\phi(t+\sigma, u)\|\right)^{p} d \sigma\right]^{1 / p}} \\
& \quad \leq\left(\left\|L_{f}\right\|_{B S^{p}}+\left\|L_{g}\right\|_{B S^{p}}\right) \epsilon+\sum_{k=1}^{m}\left(\int_{0}^{1}\left(\sup _{u \in K}\|\phi(t+\sigma, u)\|\right)^{p} d \sigma\right)^{1 / p} .
\end{aligned}
$$

For $r>r_{0}$ we have

$$
\frac{1}{m(r, \rho)} \int_{-r}^{r} \rho(t)\left(\int_{0}^{1}\left(\sup _{u \in K}\|\phi(t+\sigma, u)\|\right)^{p} d \sigma\right)^{1 / p} d t \leq\left(\left\|L_{f}\right\|_{B S^{p}}+\left\|L_{g}\right\|_{B S^{p}}+m\right) \epsilon .
$$

Hence

$$
\lim _{r \rightarrow \infty} \frac{1}{m(r, \rho)} \int_{-r}^{r} \rho(t)\left(\int_{0}^{1}\left(\sup _{u \in K}\|\phi(t+\sigma, u)\|\right)^{p} d \sigma\right)^{1 / p} d t=0 .
$$

On the other hand

$$
\begin{aligned}
& \frac{1}{m(r, \rho)} \int_{-r}^{r} \rho(t)\left\|H^{b}(t)\right\|_{q} d t \\
& \leq \frac{1}{m(r, \rho)} \int_{-r}^{r} \rho(t)\left\|H^{b}(t)\right\|_{p} d t \\
& \quad=\frac{1}{m(r, \rho)} \int_{-r}^{r} \rho(t)\left(\int_{0}^{1}\|\phi(t+\sigma, \alpha(t+\sigma))\|^{p} d \sigma\right)^{1 / p} d t \\
& \leq \frac{1}{m(r, \rho)} \int_{-r}^{r} \rho(t)\left(\int_{0}^{1}\left(\sup _{u \in K}\|\phi(t+\sigma, u)\|\right)^{p} d \sigma\right)^{1 / p} d t \rightarrow 0
\end{aligned}
$$

as $r \rightarrow \infty$. Hence $H(\cdot) \in \mathcal{B}^{-1}\left(P A A_{0}\left(\mathbb{R}, L^{q}(0,1 ; X)\right)\right)$. It proves that $f(\cdot, h(\cdot))=F(\cdot)+[G(\cdot)+$ $H(\cdot)] \in \operatorname{WPAPS}^{p}(\mathbb{R}, X)$.

The next result is the second main theorem of this paper. 
Theorem 4.10 Let $\rho \in U_{\infty}, r \geq \max \{p, p / p-1\}, \beta>0, \alpha \neq 0$, and $\alpha+\beta>0$. Suppose that:

(H1) A generates an immediately norm continuous semigroup on a Banach space $X$ and $\sup \left\{\Re \lambda, \lambda \in \mathbb{C}: \lambda(\lambda+\beta)(\lambda+\alpha+\beta)^{-1} \in \sigma(A)\right\}<0$.

(H2) The function $f=f_{1}+f_{2} \in \operatorname{WPAPS}^{p}(\mathbb{R} \times X, X)$ and there exist functions $L_{f}, L_{f_{1}} \in A P S^{r}(\mathbb{R}, \mathbb{R})$ such that

$$
\begin{aligned}
& \|f(t, u)-f(t, v)\| \leq L_{f}(t)\|u-v\|, \\
& \left\|f_{1}(t, u)-f_{1}(t, v)\right\| \leq L_{f_{1}}(t)\|u-v\|, \quad t \in \mathbb{R}, u, v \in X .
\end{aligned}
$$

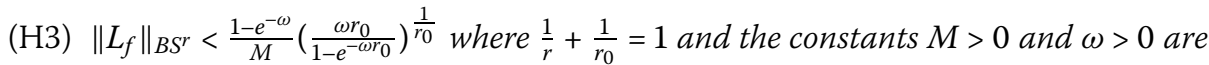
given in (4.4).

Then (4.1) has a unique mild solution in $\operatorname{WPAP}(\mathbb{R}, X)$.

Proof Let $u=u_{1}+u_{2} \in W P A P(\mathbb{R}, X)$ where $u_{1} \in A P(\mathbb{R}, X)$ and $u_{2} \in P A A_{0}(\mathbb{R}, X)$. Then $u \in$ $W_{P A P S}(\mathbb{R}, X)$. Since the range of almost periodic functions is a relatively compact set, $K=\overline{\left\{u_{1}(t): t \in \mathbb{R}\right\}}$ is compact in $X$. It follows from Theorem 4.8 that there exists $q \in[1, p)$ such that $f(\cdot, u(\cdot)) \in \operatorname{WPAPS}^{q}(\mathbb{R}, X)$. By Proposition 4.4, hypothesis (H1) implies that there exists a strongly continuous family $(S(t))_{t \geq 0}$ such that $\|S(t)\| \leq M e^{-\omega t}$ for some $M, \omega>0$.

Consider the operator $Q: \operatorname{WPAP}(\mathbb{R}, X) \rightarrow W P A P(\mathbb{R}, X)$ such that

$$
Q(u)(t):=\int_{-\infty}^{t} S(t-s) f(s, u(s)) d s \quad(t \in \mathbb{R}) .
$$

Since $f(\cdot, u(\cdot)) \in \operatorname{WPAPS}^{q}(\mathbb{R}, X)$ it follows from Theorem 3.3 that $Q$ maps $\operatorname{WPAP}(\mathbb{R}, X)$ into $\operatorname{WPAP}(\mathbb{R}, X)$.

For any $u, v \in W P A P(\mathbb{R}, X)$ we have

$$
\begin{aligned}
\|Q(u)(t)-Q(v)(t)\| & \leq \int_{-\infty}^{t}\|S(t-s)\|\|f(s, u(s)-f(s, v(s)))\| d s \\
& \leq \int_{-\infty}^{t} M e^{-\omega(t-s)} L_{f}(s)\|u(s)-v(s)\| d s \\
& \leq\|u-v\| \sum_{k=1}^{\infty} \int_{t-k}^{t-k+1} M e^{-\omega(t-s)} L_{f}(s) d s \\
& \leq\|u-v\| \sum_{k=1}^{\infty}\left(\int_{t-k}^{t-k+1} M^{r_{0}} e^{-\omega r_{0}(t-s)}\right)^{\frac{1}{r_{0}}} d s\left\|L_{f}(s)\right\|_{B S^{r}} \\
& =\frac{M}{1-e^{-\omega}}\left(\frac{1-e^{-\omega r_{0}}}{\omega r_{0}}\right)^{\frac{1}{r_{0}}}\|u-v\|\left\|L_{f}(s)\right\|_{B S^{r}} .
\end{aligned}
$$

From the Banach contraction mapping principle we find that $Q$ has a unique fixed point in $\operatorname{WPAP}(\mathbb{R}, X)$ which is the unique mild solution of (4.1).

\section{Application}

Example 5.1 Consider the problem

$$
\left\{\begin{array}{l}
\frac{\partial u}{\partial t}(t, x)=\frac{\partial^{2} u}{\partial x^{2}}(t, x)+\int_{-\infty}^{t} e^{-(t-s)} \frac{\partial^{2} u}{\partial x^{2}}(s, x) d s+f(t, u(t)) \\
u(0, t)=u(\pi, t)=0
\end{array}\right.
$$


with $x \in[0, \pi], t \in \mathbb{R}$. Let $X=L^{2}[0, \pi]$ and define $A:=\frac{\partial^{2}}{\partial x^{2}}$, with domain $D(A)=\{g \in$ $\left.H^{2}[0, \pi]: g(0)=g(\pi)=0\right\}$. Then (5.1) can be rewritten into the abstract form (4.1) with $\alpha=\beta=1$. It is well known that $A$ generates an analytic (and hence immediately norm continuous) and compact $C_{0}$-semigroup $T(t)$ on $X$. The compactness of $T(t)$ implies that $\sigma(A)=\sigma_{p}(A)=\left\{-n^{2}: n \in \mathbb{N}\right\}$. Since we must have $\lambda(\lambda+\beta)(\lambda+\alpha+\beta)^{-1} \in \sigma(A)$ we need to solve the equations $\frac{\lambda(\lambda+1)}{\lambda+2}=-n^{2}$, obtaining (see [28])

$$
\lambda_{1}=-1 \pm i, \quad \lambda_{2}=\frac{-5 \pm 7 i}{2}
$$

and

$$
\lambda_{n}=\frac{-\left(n^{2}+1\right) \pm \sqrt{\left(n^{2}-3\right)^{2}-8}}{2} \leq-2
$$

for all $n \geq 3$. We conclude that

$$
\sup \left\{\Re \lambda: \lambda(\lambda+\beta)(\lambda+\alpha+\beta)^{-1} \in \sigma(A)\right\}=-1 .
$$

Hence, from Theorem 4.7 we see that, if $f \in \operatorname{WPAPS}^{p}(\mathbb{R} \times X, X)$ satisfies

$$
\|f(t, u)-f(t, v)\| \leq L\|u-v\|
$$

for all $t \in \mathbb{R}$ and $u, v \in X$, then problem (5.1) has a unique mild solution $u \in \operatorname{WPAP}(\mathbb{R}, X)$.

Competing interests

The authors declare that they have no competing interests.

\section{Authors' contributions}

The authors contributed equally in the writing of this paper. All authors read and approved final manuscript.

\section{Author details}

${ }^{1}$ Departamento de Matemáticas, Facultad de Ciencias Básicas, Universidad del Atlántico, Barranquilla, Colombia.

${ }^{2}$ Departamento de Matemática y Ciencia de la Computación, Facultad de Ciencia, Universidad de Santiago de Chile,

Casilla 307, Correo 2, Santiago, Chile.

\section{Acknowledgements}

C Lizama is partially supported by Proyecto Anillo ACT 1112 and FONDECYT 1140258.

Received: 9 September 2014 Accepted: 13 January 2015 Published online: 31 January 2015

\section{References}

1. Diagana, T, N'Guérékata, GM, Mophou, GM: Existence of weighted pseudo almost periodic solutions to some classes of differential equations with $S^{P}$-weighted pseudo almost periodic coefficients. Nonlinear Anal. 72, 430-438 (2010)

2. Lizama, C, Ponce, R: Bounded solutions to a class of semilinear integro-differential equations in Banach spaces. Nonlinear Anal. 74, 3397-3406 (2011)

3. Prüss, J: Evolutionary Integral Equations and Applications. Monographs Math., vol. 87. Birkhäuser, Basel (1993)

4. Diagana, T: Weighted pseudo almost periodic functions and applications. C. R. Acad. Sci. Paris, Ser. I 343(10), 643-646 (2006)

5. Zhang, L, Xu, Y: Weighted pseudo almost periodic solutions for functional differential equations. Electron. J. Differ. Equ. 2007, 146 (2007)

6. Agarwal, RP, Diagana, T, Hernández, EM: Weighted pseudo almost periodic solutions to some partial neutral functional differential equations. J. Nonlinear Convex Anal. 8(3), 397-415 (2007)

7. Ezzinbi, K, Fatajou, S, N'Guérékata, GM: Weighted pseudo-almost periodic solutions for some neutral partial functional differential equations. Electron. J. Differ. Equ. 2010, 128 (2010)

8. Chen, $\mathrm{X}, \mathrm{Hu}, \mathrm{X}$ : Weighted pseudo almost periodic solutions of neutral functional differential equations. Nonlinear Anal., Real World Appl. 12(1), 601-610 (2011)

9. Diagana, T: Existence of weighted pseudo almost periodic solutions to some non-autonomous differential equations. Int. J. Evol. Equ. 2(4), 397-410 (2008) 
10. Diagana, T: Weighted pseudo-almost periodic solutions to a neutral delay integral equation of advanced type. Nonlinear Anal. 70(1), 298-304 (2009)

11. Diagana, T: Existence of weighted pseudo almost periodic solutions to some classes of hyperbolic evolution equations. J. Math. Anal. Appl. 350(1), 18-28 (2009)

12. Boukli-Hacene, N, Ezzinbi, K: Weighted pseudo almost periodic solutions for some partial functional differential equations. Nonlinear Anal. 71(9), 3612-3621 (2009)

13. Liu, J-H, Song, X-Q, Zhang, P-L: Weighted pseudo almost periodic mild solutions of semilinear evolution equations with nonlocal conditions. Appl. Math. Comput. 215(5), 1647-1652 (2009)

14. Zhang, J, Xiao, TJ, Liang, J: Weighted pseudo almost-periodic functions and applications to semilinear evolution equations. Abstr. Appl. Anal. 2012, Article ID 179525 (2012)

15. Agarwal, RP, de Andrade, B, Cuevas, C: Weighted pseudo-almost periodic solutions of a class of semilinear fractional differential equations. Nonlinear Anal., Real World Appl. 11(5), 3532-3554 (2010)

16. Cao, J, Yang, Q. Huang, Z: Optimal mild solutions and weighted pseudo-almost periodic classical solutions of fractional integro-differential equations. Nonlinear Anal. 74(1), 224-234 (2011)

17. Zhang, L, Xu, Y: Positive weighted pseudo-almost periodic solutions to infinite delay integral equation. Ann. Differ. Equ. 27(2), 253-257 (2011)

18. Zhang, L, Li, H: Weighted pseudo almost periodic solutions of second order neutral differential equations with piecewise constant argument. Nonlinear Anal. 74(17), 6770-6780 (2011)

19. Zhang, L, Li, H: Weighted pseudo almost periodic solutions of second-order neutral-delay differential equations with piecewise constant argument. Comput. Math. Appl. 62(12), 4362-4376 (2011)

20. Ding, HS, N'Guérékata, GM, Nieto, Jj: Weighted pseudo almost periodic solutions for a class of discrete hematopoiesis model. Rev. Mat. Complut. 26(2), 427-443 (2013)

21. Bochner, S: Beiträge zur theorie der fastperiodischen funktionen. Math. Ann. 96, 119-147 (1927)

22. Bochner, S: A new approach to almost automorphicity. Proc. Natl. Acad. Sci. USA 48, 907-910 (1964)

23. Chang, YK, N'Guérékata, GM, Zhang, R: New composition theorems of Stepanov-like weighted pseudo almost automorphic functions and applications to nonautonomous evolution equations. Nonlinear Anal., Real World Appl. $13,2866-2879(2012)$

24. Diagana, T: Stepanov-like pseudo almost periodic functions and their applications to differential equations. Commun. Math. Anal. 3(1), 9-18 (2007)

25. Alkahby, H, Lee, H: Stepanov-like almost automorphic solutions of nonautonomous semilinear evolution equations with delay. Nonlinear Anal. 69, 2158-2166 (2008)

26. N'Guérékata, GM, Pankov, A: Stepanov-like almost automorphic functions and monotone evolution equations. Nonlinear Anal. 68, 2658-2667 (2008)

27. Ding, H, Liang, J, Xiao, TJ: Weighted pseudo almost automorphic functions and WPAA solutions to semilinear evolution equations. J. Math. Anal. Appl. 409, 409-427 (2014)

28. Chen, J, Liang, J, Xiao, TJ: Uniform exponential stability of solutions to abstract Volterra equations. J. Evol. Equ. 9(4), 661-674 (2009)

29. Long, W, Ding, H: Composition theorems of Stepanov almost periodic functions and Stepanov-like pseudo almost periodic functions. Adv. Differ. Equ. 2011, Article ID 654695 (2011). doi:10.1155/2011/654695

\section{Submit your manuscript to a SpringerOpen ${ }^{\odot}$ journal and benefit from:}

- Convenient online submission

Rigorous peer review

Immediate publication on acceptance

Open access: articles freely available online

- High visibility within the field

- Retaining the copyright to your article 